\title{
Trazabilidad de los discursos sobre tecnología educativa: los caminos de la influencia
}

\author{
Educational Technology Discourses Traceability: The Influence Paths
}

\author{
Linda Castañeda \\ Universidad de Murcia (España) \\ lindacq@um.es
}

\section{CITA RECOMENDADA}

Castañeda, L. (2021). Trazabilidad de los discursos sobre tecnología educativa: los caminos de la influencia. Revista Interuniversitaria de Investigación en Tecnología Educativa, 10, 1-8. https://doi.org/10.6018/riite.480011

\section{INTRODUCCIÓN}

Desde hace más de dos décadas, la inclusión de la tecnología digital en las prácticas educativas ha sido percibida por una parte de los miembros de la comunidad educativa como una necesidad esencial. Buena prueba de ello es la extensa producción de discursos y políticas que recomiendan, fomentan y regulan su uso (Beetham \& Sharpe, 2013; Buckingham et al., 2001; Cabero, 2016; Salinas, 2016). Sin embargo, la crisis social provocada por la pandemia Covid19 , los confinamientos, los problemas educativos asociados a las prácticas escolares digitales a la llamada "educación remota de emergencia"-, y, en última instancia, la exposición generalizada a la realidad de lo digital en educación, han concienciado a muchas personas de la dificultad y de la importancia de sacar provecho de la tecnología en el mundo de la educación.

En primer lugar, porque hemos sido conscientes de la importancia de la generalización del uso de la tecnología en la educación (Betancor et al., 2021; Bozkurt et al., 2020; Daniel, 2020; Gourlay, 2020; Onyema et al., 2020); en segundo lugar porque se ha revelado por fin la magnitud del reto que ese "uso de las TIC" supone en la educación actual no solo en términos logísticos o tecnológicos, sino en términos de pedagogía, diversidad, igualdad y hasta de estrategia económica (Czerniewicz, 2020; Williamson \& Hogan, 2021). Finalmente, porque todo esto ha traído consigo la necesidad de entender a qué concepto de tecnología educativa y de educación responden nuestras prácticas, es decir cómo y por qué hacemos lo que hacemos con la tecnología en las instituciones educativas (Biesta et al., 2020a; Regan \& Khwaja, 2019; Rensfeldt \& Player-Koro, 2020; Selwyn et al., 2020).

Como hemos dicho, estas cuestiones no son nuevas. Algunas ya habían sido abordadas y reiteradamente discutidas por aquellos que se dedican desde hace tiempo a la educación en general y a la tecnología educativa en particular. Sin embargo, existe un asunto que no ha sido abordado suficientemente, al menos desde perspectivas críticas, sistemáticas y a escala suficiente: la forma en que los discursos sobre tecnología educativa se generan, traducen, difunden, transforman, se apropian y materializan en la realidad educativa, o lo que es lo mismo, los mecanismos de influencia educativa relacionados con los discursos sobre tecnología educativa (en adelante DTE).

La tecnología interviene en la educación de formas diversas, tanto como campo de estudio -la tecnología educativa-, como como sector económico -el sector EdTech del que tanto se habla-, y preocupa a la sociedad hasta el punto de ser foco de iniciativas políticas y sociales en casi todos los niveles (Caena, 2014; Selwyn, 2018). Sin embargo, todavía sabemos poco sobre cómo los discursos que traen consigo esas iniciativas de tecnología educativa -y que incluyen premisas y supuestos sobre la relación entre el ser humano y la sociedad con la tecnología, las lógicas del desarrollo tecnológico, los enfoques epistémicos que asume, la organización escolar 
que se deriva de ellos, el modelo de docente (y de su formación inicial y permanente) que promueve, la política educativa que se deriva de ellos, etc.- interactúan e impactan en la realidad de las aulas (Biesta et al., 2020b; Cerratto-Jahnke \& Jahnke, 2019; Goodyear, 2020).

La llegada de las herramientas digitales a la escuela vino acompañada de muchas promesas: mejora de la calidad, incremento de la igualdad, acercamiento social, pensamiento crítico, democratización del acceso, colaboración, etc. (Anderson \& Rivera-Vargas, 2020). Sin embargo, muchas de esas promesas no se han cumplido. De hecho, muchas de esas cuestiones, como la democratización y el pensamiento crítico, lejos de mejorar, han empeorado (Castañeda, 2019; Castañeda \& Selwyn, 2018; Selwyn, 2018). Entender la racionalidad detrás de esas esperanzas y la causa de su incumplimiento implica un conocimiento profundo y un rastreo complejo de los discursos que las soportan, los DTE.

En esta reflexión partimos de la idea de que entender los DTE y los mecanismos que operan con ellos resulta crucial para comprender cómo la tecnología -y las realidades asociadas a ellainfluye en la educación en general y en la didáctica en particular, cuando no las determinan. Sin embargo, esa influencia no es lineal ni mucho menos simple, como casi nada en educación y viene marcada por una realidad multifactorial y compleja que la condiciona. Por ello he considerado relevante detenerme y reflexionar sobre algunos de los cambios de los últimos años que hacen más urgente si cabe esa comprensión, y pretendo desde ellos formular, al menos de forma tentativa, algunas líneas de desarrollo que podrían plantearse de cara al futuro inmediato y a largo plazo en términos de investigación y debate.

\section{FUENTES DE INFLUENCIA CONTEMPORÁNEAS}

La tecnología educativa es un campo de investigación y de práctica en el que confluyen actores e intereses diversos (Castañeda \& Williamson, 2021; Livingston, 2018; Tahirsylaj, 2019) Cada uno de ellos persigue sus intereses promoviendo diversos tipos de DTE.

De forma transversal, los medios de comunicación masivos, los grandes comunicadores de redes sociales (los influencers de lo educativo o lo tecnológico o de ambos), e incluso los usuarios comprometidos avanzados (profesores-"embajadores", o "certificados" por una o varias empresas tecnológicas) inundan las redes sociales con mensajes de palabras clave, que relacionan íntimamente tecnología y educación y que lo envuelven todo (Flipped, Personal, Digital o Data Literacies, Computational Thinking, Adaptive, Algorithmic, Inteligent, Smart, etc.) (Selwyn, 2016). La mayoría de estos términos, a pesar de su poca definición y claridad, aparecen mencionadas como "clave" e "indispensables" para la mejora de la educación y son lugares comunes que no pueden faltar en discursos académicos, programas de desarrollo profesional docente, informes futuristas, estudios de investigación y en ofertas comerciales de editoriales y empresas tecnológicas (Bridgstock, 2016; Player-Koro et al., 2018).

El profesorado por su parte intenta traducir e incluir algunos de estos nuevos discursos alrededor de imaginarios sociotécnicos en sus prácticas y en sus diseños didácticos (Jasanoff \& Kim, 2015; Rensfeldt \& Player-Koro, 2020; Spiteri \& Chang Rundgren, 2020), aunque el camino entre la palabra incluida (mediado por quién la incluye y la "traduce") y la materialización de sus consecuencias en la realidad educativa está, digamos, muy poco clara.

Esas palabras de moda no solo suponen anécdotas en el vocabulario. Tras ellas, a pesar de su poca definición, suelen acogerse y generarse discursos enteros sobre educación, algunos explícitos y otros más o menos velados. La cuestión es que la creación y difusión de esas palabras de moda, generadoras de DTE, no se circunscribe a investigadores, teóricos o prácticos de la educación, sino que en muchos casos proviene de fuera de sectores con un "interés educativo" claro, lo cual puede suponer un problema de conflicto de intereses que no siempre es evidente. El mercado y la industria creados en torno a la educación, y en concreto a la tecnología educativa (el llamado sector EdTech), se ha revelado en los últimos 10 años como uno de los grandes valores emergentes del mercado a nivel mundial que moviliza miles de millones de euros al año (Gran View Research, 2020; HolonIQ, 2020). En ese contexto es un reto diferenciar las prioridades educativas de otras prioridades, y los mensajes que provienen de la industria no terminan de explicitar qué lado están (Munro, 2018; Verger et al., 2017; Watters, 2016). Esa escasa definición, o ese conflicto de intereses que parece no resuelto, ha sido señalado como 
uno de los mayores problemas a la hora de desentrañar la cuestión de cómo los DTE se materializan en la realidad educativa (Castañeda \& Williamson, 2021; Honan, 2010; Perrotta et al., 2020; Regan \& Khwaja, 2019).

Además nos encontramos con un extenso espacio de información copado por documentos transnacionales, encargados por empresas, asociaciones y colectivos variados, que con la declarada intención de tratar de mapear el porvenir inmediato de la educación, presentan informes sobre un futuro inmediato, a medio y largo plazo de la innovación educativa (paradigmáticos los informes Horizon, primero promovidos por el New Media Consortium y ahora asumidos por EDUCAUSE (Alexander et al., 2019), o el Innovating Pedagogies, firmado por la Open University de Reino Unido (Kukulska-Hulme et al., 2021), pero hay muchos más). En estos informes, de los que se han hecho análisis demoledores en algún caso (Watters, 2017), se da la paradoja de que sus predicciones se basan en metodologías que recogen la opinión de expertos del ámbito académico y sirven, a la vez, como justificación en una cantidad ingente de investigaciones y artículos sobre innovación educativa y tecnología. Lo cual genera un bucle de influencia en el que no termina de estar claro si muestran tendencias, crean tendencias, crean burbujas de tendencias, o todo a la vez, quién se ve influido por esas predicciones y, sobre todo, a quién sirven.

Por otro lado, es relevante remarcar la influencia cada vez menos encubierta de actores externos al sistema educativo en la toma de decisiones sobre políticas educativas públicas o de instituciones públicas. Así, las autoridades oficiales (políticas, educativas), académicas y los actores con intereses "estrictamente educativos" son solo una pequeña parte del espectro de implicados que influyen de forma más o menos directa en la generación de discursos y políticas relacionadas con la tecnología (Spring, 2012). Incluso en términos estatales y supra-nacionales, muchas redes o comunidades de actores antes considerados "externos" desempeñan un papel fundamental como referentes, participantes y fuentes de influencia en las políticas sobre implementación de tecnología educativa, a pesar de su falta de legitimidad democrática o siquiera intelectual (Menashy, 2015; Stone, 2008). Este tipo de influencias han sido particularmente fuertes y obvias en tiempos del CoVid-19, donde la pandemia ha sido una oportunidad de negocio y el discurso sobre la adopción e implementación tecnológica de modelos educativos "híbridos", por ejemplo, un envoltorio legitimador de prácticas que no está claro cómo son de beneficiosas en términos educativos más allá del confinamiento, y cuyas consecuencias generan la impresión de que el uso de las tecnologías y las políticas derivadas del mismo durante la crisis global CoVid-19 haya sido tan necesario e inevitable, como traumático. Algunos autores han calificado lo ocurrido como "procesos de privatización encubierta" (Bozkurt et al., 2020; Williamson \& Hogan, 2021).

Finalmente, es fundamental ser conscientes de que la influencia de los nuevos actores de la tecnología educativa y de sus discursos ha transformado los entornos de aprendizaje y desarrollo profesional de los educadores. Por un lado, nos encontramos con esfuerzos corporativos que aglutinan o definen comunidades de intercambio, enseñanza y entrenamiento para educadores de todos los niveles; espacios que vienen avalados mayoritariamente por empresas tecnológicas, plataformas educativas comerciales o por grupos de presión políticoeconómicos. Esos grupos generan acuerdos de formación directa con las instituciones educativas, pero además ofertan experiencias de formación presencial y online que aglutinan gran cantidad de público y de oferta comercial. Algunos de esos espacios de formación que antes eran contextos tradicionales de aprendizaje entre pares (es decir, los congresos y encuentros entre docentes) son hoy en día espacios de promoción y de autopromoción de cierto profesorado -las "celebridades"- (Honan, 2010; Player-Koro et al., 2017) que ejercen su particular influencia asumiendo el rol de influenciadores, microinfluenciadores o embajadores, ya sea de forma independiente o "captados" por unas determinadas empresas, y jerarquizando artificialmente la profesión (Brown \& Stevick, 2014; Gu et al., 2019; Saldaña et al., 2019). Huelga decir que la forma en que estos espacios y actores (y otros específicos como las publicaciones de divulgación) mueven los DTE está afectando definitivamente a la forma en que el profesorado aprende, se desarrolla profesionalmente y está influyendo en qué prácticas de enseñanza se promueven (Biesta et al., 2020b; Stech et al., 2020).

Capítulo aparte merecen seguramente otros entes de influencia en los DTE, particularmente las líneas de investigación prioritaria de los organismos de financiación nacionales e 
internacionales que suelen marcar claramente la agenda de lo que merece o no ser investigado y que dejan a su suerte temas que seguramente pueden ser más relevantes en términos educativos pero que, por su nulo interés de financiación, son abandonados a su suerte y se convierten en prácticamente marginales.

\section{EXPLICITAR LOS MECANISMOS DE INFLUENCIA EN LOS DTE}

Los procesos de utilización de la tecnología en la educación o directamente de digitalización educativa están enmarañados y por ello muchas cuestiones que acompañan a la tecnología y a la apropiación de los DTE en las prácticas docentes aún no han sido abordadas satisfactoriamente.

Los mecanismos de influencia educativa relacionados con DTE no solo afectan a la creación de nuevos términos, más o menos vacíos. Cuando hablamos de DTE en este trabajo aludimos tanto al conocimiento académico sobre tecnologías en educación (la Tecnología Educativa), como a la publicidad del sector industrial y comercial de las tecnologías para la educación (EdTech), como a las premisas que subyacen a las prácticas educativas que implementan tecnología de una forma $u$ otra y a todos los discursos y mecanismos intermedios. Y si bien somos conscientes de que no son cosas idénticas, sí sabemos que su impacto puede ser similar.

Así, los mecanismos de influencia de los discursos -y los actores que los protagonizan- (las policy networks de las que habla Ball_(2013). tienen un impacto particularmente alto en la difusión, traducción y formulación de ideas educativas, como han señalado a lo largo de los últimos 30 años diversos autores (Granovetter, 1983; Mintrom \& Vergari, 1998; Rogers, 2003). Los roles asumidos por los "emprendedores" de una u otra "innovación", pueden cambiar la forma en que una idea específica se difunde, se traduce y se materializa, influye, media, se resiste 0 directamente se malinterpreta o se olvida (Ball, 2016).

Es decir, no se trata de desvelar solamente la representación o la visión general sobre la realidad del uso de las TIC en las aulas, sino de analizar el discurso en torno a esa representación (su naturaleza, sus consecuencias), su proveniencia y la forma en que se ha difundido y expandido. Se trata no solo de entender si esas influencias impactan positivamente en la actividad de las escuelas, en los diseños didácticos y en la actividad de aprendizaje de los estudiantes y entender los mecanismos que soportan esta influencia, sino en cuáles podrían ser mecanismos efectivos para difundir y enriquecer otros procesos de adopción de discursos.

Necesitamos abordar la trazabilidad de los DTE de manera eficiente, es decir, deberíamos ser capaces de saber cuáles son los caminos y los mecanismos que mueven determinados discursos. Siendo cada vez más conscientes de la complejidad de la práctica docente y del rol que ejercen las creencias, los conocimientos, las condiciones del profesorado en su assemblage (Strom, 2015), es preciso entender mejor los mecanismos que las influencian. Eso implica conocer, entre otras cosas: (1) cómo las ideas, creencias, metodologías y DTE impactan efectivamente en las prácticas de enseñanza y en el diseño del aprendizaje; (2) cómo los mecanismos de información operan e influyen en los actores y recursos mediáticos relacionados con los DTE, crean vías de influencia en las prácticas de enseñanza; (3) cómo los DTE proporcionados por las instituciones (teoría, políticas, investigación, etc.) está siendo traducidos por los actores influyentes; o (4) cómo esos DTE son transformados y materializados a su vez por los profesores y de qué forma están contribuyendo a la práctica diaria de las aulas.

Se trata de dejar de obviar el hecho de que todas las actuaciones emprendidas por un actor o grupo de actores en el sistema educativo, también en lo que se refiere a la tecnología educativa, responden a formas de entender educación, tecnología y la relación entre ambas, que son traducidas y asumidas de forma más o menos consciente por parte de aquellos que las usan, pero que en muchos casos han sido cuidadosamente "empaquetadas" por otros actores que ejercen así su influencia en el sistema. 


\section{REFERENCIAS BIBLIOGRÁFICAS}

Alexander, B., Ashford-Rowe, K., Barajas-Murphy, N., Dobbin, G., Knott, J., McCormack, M., Pomerantz, J., Seilhamer, R., \& Weber, N. (2019). EDUCAUSE Horizon Report: 2019 Higher Education Edition (Horizon Report, p. 44). Educause.

Anderson, T., \& Rivera-Vargas, P. (2020). A Critical look at Educational Technology from a Distance Education Perspective. Digital Education Review, O(37), 208-229. https://doi.org/10.1344/der.2020.37.208-229

Ball, S. (2013). The education debate. Policy Press.

Ball, S. (2016). Following policy: Networks, network ethnography and education policy mobilities. Journal of Education Policy, 31(5), 549-566. https://doi.org/10.1080/02680939.2015.1122232

Beetham, H., \& Sharpe, R. (2013). Rethinking Pedagogy for a Digital Age. Routledge. Taylor \& Francis Group.

Betancor, G., María, S., Puig, L., Jorge, A., \& Eugenia, C. (2021). Digital inequality at home. The school as compensatory agent. Computers \& Education, 104195. https://doi.org/10.1016/j.compedu.2021.104195

Biesta, G., Takayama, K., Kettle, M., \& Heimans, S. (2020a). Call for papers: A new agenda for teacher education research. Asia-Pacific Journal of Teacher Education, 48(5), 460-462. https://doi.org/10.1080/1359866X.2020.1818484

Biesta, G., Takayama, K., Kettle, M., \& Heimans, S. (2020b). Teacher education between principle, politics, and practice: A statement from the new editors of the Asia-Pacific Journal of Teacher Education. Asia-Pacific Journal of Teacher Education, 48(5), 455-459. https://doi.org/10.1080/1359866X.2020.1818485

Bozkurt, A., Jung, I., Xiao, J., Vladimirschi, V., Schuwer, R., Egorov, G., Lambert, S., AlFreih, M., Pete, J., Don Olcott, J., Rodes, V., Aranciaga, I., Bali, M., Alvarez, A. J., Roberts, J., Pazurek, A., Raffaghelli, J. E., Panagiotou, N., Coëtlogon, P. de, ... Paskevicius, M. (2020). A global outlook to the interruption of education due to COVID-19 pandemic: Navigating in a time of uncertainty and crisis. Asian Journal of Distance Education, 15(1), 1-126.

Bridgstock, R. (2016). Educating for digital futures: What the learning strategies of digital media professionals can teach higher education. Innovations in Education and Teaching International, 53(3), 306-315. https://doi.org/10.1080/14703297.2014.956779

Brown, K. D., \& Stevick, D. (2014). The Globalizing Labor Market in Education: Teachers as Cultural Ambassadors or Agents of Institutional Isomorphism? Peabody Journal of Education, 89(1), 4-16. https://doi.org/10.1080/0161956X.2014.862134

Buckingham, D., Scanlon, M., \& Sefton-Green, J. (2001). Selling the Digital Dream. Marketing educational technology to teachers and parents. In A. M. Loveless \& V. Ellis (Eds.), ICT, Pedagogy and the Curriculum: Subject to Change. Routledge.

Cabero, J. (2016). ¿Qué debemos aprender de las pasadas investigaciones en Tecnología Educativa? Revista Interuniversitaria de Investigación en Tecnología Educativa, O(0). http://revistas.um.es/riite/article/view/256741

Caena, F. (2014). Teacher Competence Frameworks in Europe: Policy-as-discourse and policy-as-practice. European Journal of Education, 49(3), 311-331. https://doi.org/10.1111/ejed.12088

Castañeda, L. (2019). Debates sobre Tecnología y Educación: Caminos contemporáneos y conversaciones pendientes. RIED. Revista Iberoamericana de Educación a Distancia, 22(1). https://doi.org/10.5944/ried.22.1.23020

Castañeda, L., \& Selwyn, N. (2018). More than tools? Making sense of the ongoing digitizations of higher education. International Journal of Educational Technology in Higher Education, 15, 22. https://doi.org/10.1186/s41239-018-0109-y 
Castañeda, L., \& Williamson, B. (2021). Assembling New Toolboxes of Methods and Theories for Innovative Critical Research on Educational Technology. Journal of New Approaches in Educational Research, 10(1), 1-14. https://doi.org/10.7821/naer.2021.1.703

Cerratto-Jahnke, T., \& Jahnke, I. (Eds.). (2019). Emergent Practices and Material Conditions in Learning and Teaching with Technologies. Springer International Publishing. https://doi.org/10.1007/978-3-030-10764-2

Czerniewicz, L. (2020, March 15). What we learnt from "going online" during university shutdowns in South Africa. PhilOnEdTech. https://philonedtech.com/what-we-learnt-from-goingonline-during-university-shutdowns-in-south-africa/

Daniel, S. J. (2020). Education and the COVID-19 pandemic. PROSPECTS, 49(1), 91-96. https://doi.org/10.1007/s11125-020-09464-3

Goodyear, P. (2020). Design and co-configuration for hybrid learning: Theorising the practices of learning space design. British Journal of Educational Technology, 51(4), 1045-1060. https://doi.org/10.1111/bjet.12925

Gourlay, L. (2020). Quarantined, Sequestered, Closed: Theorising Academic Bodies Under Covid-19 Lockdown. Postdigital Science and Education, 1-21. https://doi.org/10.1007/s42438020-00193-6

Gran View Research. (2020). Education Technology Market Size Report, 2020-2027 (GVR4-68038-878-7). Gran View Research. https://www.grandviewresearch.com/industryanalysis/education-technology-market

Granovetter, M. (1983). The Strength of Weak Ties: A Network Theory Revisited. Sociological Theory, 1, 201-233. https://doi.org/10.2307/202051

Gu, X., Crook, C., \& Spector, M. (2019). Facilitating innovation with technology: Key actors in educational ecosystems. British Journal of Educational Technology, 50(3), 1118-1124. https://doi.org/10.1111/bjet.12786

HolonIQ. (2020, August 6). Global EdTech market to reach $\$ 404 \mathrm{~B}$ by 2025-16.3\% CAGR. [HolonIQ]. Holon/Q. https://www.holoniq.com/notes/global-education-technology-market-toreach-404b-by-2025/

Honan, E. (2010). Mapping discourses in teachers' talk about using digital texts in classrooms. Discourse: Studies in the Cultural Politics of Education, 31(2), 179-193. https://doi.org/10.1080/01596301003679701

Jasanoff, S., \& Kim, S.-H. (2015). Dreamscapes of Modernity: Sociotechnical Imaginaries and the Fabrication of Power. University of Chicago Press. https://doi.org/10.7208/chicago/9780226276663.001.0001

Kukulska-Hulme, A., Bossu, C., Coughlan, T., Ferguson, R., FitzGerald, E., Gaved, M., Herodotou, C., Rienties, B., Sargent, J., Scanlon, E., Tang, J., Wang, Q., Whitelock, D., \& Zhang, S. (2021). Innovating Pedagogy 2021 (p. 55). Open University, UK.

Livingston, K. (2018). Multiple influences on teachers in changing environments. European Journal of Teacher Education, 41(2), 135-137. https://doi.org/10.1080/02619768.2018.1432318

Menashy, F. (2015). Understanding the roles of non-state actors in global governance: Evidence from the Global Partnership for Education. Journal of Education Policy, 31(1), 98-118. https://doi.org/10.1080/02680939.2015.1093176

Mintrom, M., \& Vergari, S. (1998). Policy Networks and Innovation Diffusion: The Case of State Education Reforms. The Journal of Politics, 60(1), 126-148. https://doi.org/10.2307/2648004

Munro, M. (2018). The complicity of digital technologies in the marketisation of UK higher education: Exploring the implications of a critical discourse analysis of thirteen national digital teaching and learning strategies. International Journal of Educational Technology in Higher Education, 15(1), 11. https://doi.org/10.1186/s41239-018-0093-2 
Onyema, E. M., Eucheria, N. C., Obafemi, F. A., Sen, S., Atonye, F. G., Sharma, A., \& Alsayed, A. O. (2020). Impact of Coronavirus Pandemic on Education. Journal of Education and Practice, 11(13), 108.

Perrotta, C., Gulson, K. N., Williamson, B., \& Witzenberger, K. (2020). Automation, APIs and the distributed labour of platform pedagogies in Google Classroom. Critical Studies in Education, O(0), 1-17. https://doi.org/10.1080/17508487.2020.1855597

Player-Koro, C., Bergviken Rensfeldt, A., \& Selwyn, N. (2017). Selling tech to teachers: Education trade shows as policy events. Journal of Education Policy, 1-22. https://doi.org/10.1080/02680939.2017.1380232

Player-Koro, C., Rensfeldt, A. B., \& Selwyn, N. (2018). Selling tech to teachers: Education trade shows as policy events. Journal of Education Policy, 33(5), 682-703. https://doi.org/10.1080/02680939.2017.1380232

Regan, P. M., \& Khwaja, E. T. (2019). Mapping the political economy of education technology: A networks perspective. Policy Futures in Education, 17(8), 1000-1023. https://doi.org/10.1177/1478210318819495

Rensfeldt, A. B., \& Player-Koro, C. (2020). "Back to the future": Socio-technical imaginaries in 50 years of school digitalization curriculum reforms. Seminar.Net, 16(2), 20-20. https://doi.org/10.7577/seminar.4048 Schuster

Rogers, E. M. (2003). Diffusion of Innovations, 5th Edition (Edición: 5th Edition.). Simon \&

Saldaña, C. M., Welner, K. G., Malcolm, S., \& Tisch, E. (2019). Examining the New Phenomenon of Teachers as Brand Ambassadors. In Commercialism in Education Research Unit. Commercialism in Education Research Unit. https://eric.ed.gov/?id=ED593277

Salinas, J. (2016). La investigación ante los desafíos de los escenarios de aprendizaje futuros. Revista de Educación a Distancia (RED), 50. https://doi.org/10.6018/red/50/13

Selwyn, N. (2016). Is technology good for education? Polity Press.

Selwyn, N. (2018). Technology as a Focus of Education Policy. In The Wiley Handbook of Educational Policy (pp. 457-477). John Wiley \& Sons, Ltd. https://doi.org/10.1002/9781119218456.ch20

Selwyn, N., Hillman, T., Eynon, R., Ferreira, G., Knox, J., Macgilchrist, F., \& Sancho-Gil, J. M. (2020). What's next for Ed-Tech? Critical hopes and concerns for the 2020s. Learning, Media and Technology, 45(1), 1-6. https://doi.org/10.1080/17439884.2020.1694945

Spiteri, M., \& Chang Rundgren, S.-N. (2020). Literature Review on the Factors Affecting Primary Teachers' Use of Digital Technology. Technology, Knowledge and Learning, 25(1), 115128. https://doi.org/10.1007/s10758-018-9376-x

Spring, J. (2012). Globalization of Education. International Journal of Chinese Education, 1(2), 139-176. https://doi.org/10.1163/22125868-12340002

Stech, S., Šed'ová, K., \& Lefstein, A. (2020). What is happening to the teaching profession? Editorial. Studia Paedagogica, 25(2), 5-10.

Stone, D. (2008). Global Public Policy, Transnational Policy Communities, and Their Networks. Policy Studies Journal, 36(1), 19-38. https://doi.org/10.1111/j.15410072.2007.00251.x

Strom, K. J. (2015). Teaching as Assemblage. Journal of Teacher Education, 66(4), 321333. https://doi.org/10.1177/0022487115589990

Tahirsylaj, A. (2019). Revisiting 'curriculum crisis' dialogue: In search of an antidote. Nordic Journal of Studies in Educational Policy, 5(3), 180-190. https://doi.org/10.1080/20020317.2019.1664217 
Verger, A., Steiner-Khamsi, G., \& Lubienski, C. (2017). The emerging global education industry: Analysing market-making in education through market sociology. Globalisation, Societies and Education, 15(3), 325-340. https://doi.org/10.1080/14767724.2017.1330141

Watters, A. (2016). The Curse of the Monsters of Education Technology. Amazon.

Watters, A. (2017, February 16). What's on the Horizon (Still, Again, Always) for Ed-Tech. Hack Education. http://hackeducation.com/2017/02/16/horizon

Williamson, B., \& Hogan, A. (2021). Pandemic Privatisation in Higher Education: Edtech \& University Reform. Education International. https://www.eiie.org/en/detail/17124/\%E2\%80\%9Cpost-pandemic-reform-of-higher-education-market-first-orpurpose-first-digital-transformation\%E2\%80\%9D-by-ben-williamson-and-anna-hogan

\section{INFORMACIÓN SOBRE LA AUTORA}

\section{Linda Castañeda}

Universidad de Murcia

Linda Castañeda es Licenciada en Pedagogía por la Universidad de Murcia y Doctora en Tecnología Educativa por la Universidad de les Illes Balears. Profesora Titular de Universidad del Departamento de Didáctica y Organización Escolar de la Facultad de Educación de la Universidad de Murcia y miembro del Grupo de Investigación de Tecnología Educativa de la misma Universidad. Su investigación se interesa especialmente por el impacto de la tecnología en la educación a todos los niveles, el análisis crítico de la Tecnología Educativa, la implementación de estrategias didácticas emergentes en contextos formales y no formales, los Entornos Personales de Aprendizaje (PLE) y el desarrollo de competencias para el mundo digital. Más información sobre el trabajo y publicaciones en www.lindacastaneda.com

ORCID https://orcid.org/0000-0002-1055-9241

\footnotetext{
(c) $)(90$

Los textos publicados en esta revista están sujetos a una licencia de Reconocimiento 4.0 España de Creative Commons. Puede copiarlos, distribuirlos, comunicarlos públicamente y hacer obras derivadas siempre que reconozca los créditos de las obras (autoría, nombre de la revista, institución editora) de la manera especificada por los autores o por la revista. La licencia completa se puede consultar en:Licencia Creative Commons Atribución-NoComercial-Compartir por igual 4.0 Internacional.
} 